\title{
Developing Technologies for Biological Experiments in Deep Space ${ }^{+}$
}

\author{
Elizabeth M. Hawkins 1,2,3, Ada Kanapskyte ${ }^{1,4}$ and Sergio R. Santa Maria 5,6,* \\ 1 Space Life Sciences Training Program, NASA Ames Research Center, Moffett Field, CA 94035, USA; \\ 2 KBR Wyle, Moffett Field, CA 94035, USA \\ 3 Mammoth Biosciences, Inc., South San Francisco, CA 94080, USA \\ 4 Biomedical Engineering Department, The Ohio State University, Columbus, OH 43210, USA \\ Space Biosciences, NASA Ames Research Center, Moffett Field, CA 94035, USA \\ 6 COSMIAC Research Institute, University of New Mexico, Albuquerque, NM 87131, USA \\ * Correspondence: sergio.santamaria@nasa.gov; Tel.: +1-650-604-1411 \\ + Presented at the 1st International Electronic Conference on Biosensors, 2-17 November 2020; Available \\ online: https://iecb2020.sciforum.net/.
}

Published: date

\begin{abstract}
In light of an upcoming series of missions beyond low Earth orbit (LEO) through NASA's Artemis program and the potential establishment of bases on the Moon and Mars, the effects of the deep space environment on biology need to be examined and protective countermeasures need to be developed. Even though many biological experiments have been performed in space since the 1960s, most of them have occurred in LEO for short periods of time. These missions have studied many biological phenomena in a variety of model organisms, as well as utilized a broad range of technologies. However, given the constraints of the deep space environment, future planned biological missions will be limited to microbial organisms using miniaturized technologies. Small satellites like CubeSats are capable of querying relevant space environments using novel instruments and biosensors. CubeSats also provide a low-cost alternative to more complex and larger missions, and require minimal crew support, if any. Several have been deployed in LEO, but the next iteration of biological CubeSats will go farther. BioSentinel will be the first interplanetary CubeSat and the first biological study NASA has sent beyond Earth's magnetosphere in 50 years. BioSentinel is an autonomous free-flyer platform able to support biology and to investigate the effects of radiation on a model organism in interplanetary deep space. Aside from this objective, the BioSensor payload contained within the free-flyer is also an adaptable instrument that can perform biologically relevant measurements with different microorganisms and in multiple space environments, including the ISS, lunar gateway, and on the surface of the Moon. Thus, nanosatellites like BioSentinel can be used to study the effects of both reduced gravity and space radiation and can house different organisms or biosensors to answer specific science questions. Utilizing these biosensors will allow us to better understand the effects of the space environment on biology so humanity may return safely to deep space and venture farther than ever before.
\end{abstract}

Keywords: deep space; biosensors; space radiation; microgravity; CubeSat; BioSentinel 


\section{Introduction}

NASA currently has plans to return humans to the Moon and to eventually land manned missions on Mars. This goal is unachievable unless we can ensure the safety and health of the astronaut crew and other terrestrial biology on those missions. The last time NASA sent biology beyond low Earth orbit (LEO) was during the last Apollo mission in 1972. Since then, long-duration missions have been confined to LEO, such as those to the International Space Station (ISS).

The deep space environment is characterized by reduced gravity and ionizing radiation in the form of galactic cosmic rays (GCRs) and solar particle events (SPEs), both of which can have detrimental effects on biology. In humans, it is well known that microgravity induces health risks such as muscle atrophy and bone density loss. It is less well known that reduced gravity can have effects at the subcellular level, affecting gene expression and cell growth pathways [1]. In plants, a phenomenon called gravitropism causes roots to grow downward, but in space, their roots grow randomly [2]. Additionally, many bacteria have been shown to display increased virulence and antibiotic resistance when exposed to the space environment [3].

The space radiation environment is a cocktail of dangerous ionizing particles. Beyond the Earth's magnetosphere, biology will be exposed to a constant, low-flux shower of high-energy, ionizing radiation, such as those from GCRs and SPEs. Ionizing radiation causes damage to biology through several means, including direct DNA damage like double-strand breaks and indirect damage like that caused by reactive oxygen species [4].

It is critical for the future of space exploration that more biological studies be conducted querying the deep space environment. However, it is expensive and dangerous to send humans to space, and readouts for the effects of the space environment on humans is complicated. A method of simplifying biological experiments is by using model organisms. NASA has performed many biological missions utilizing model organisms within LEO since 1972, as will be discussed. Higherorder, multicellular eukaryotes are complex, but glean more human-relevant information. They also often require complicated and bulky technology. While many space experiments have been performed in higher eukaryotes including rodents and other animal models, current planned missions beyond LEO will include single-celled prokaryotes and eukaryotes and may include extremophiles and microbial communities. Benefits of microbes include that they require minimal care and interaction, and biological and biochemical assays often have straightforward and quantifiable readouts.

Combining microbes with the miniaturization and automation of new technologies, it is possible to perform highly sophisticated experiments. Biological experiments in space require very specialized hardware, such as microfluidics and detection sensors, as well as reliable automation and data handling. Small satellites known as CubeSats are instrumental platforms that can accommodate these requirements and can be used to answer questions about the effects of the space environment on biology. In recent years, microbial-derived biosensors aboard CubeSats have been used, for example, to investigate the effect of microgravity on antibiotic resistance in pathogenic bacteria and to study the effect of a fungicide on yeast cells $[5,6]$. These recent studies have been built on a foundation of decades of space biology research.

\section{Past and Current Technologies}

To fully understand the role of biosensors in space research, it is helpful to reflect upon a timeline of NASA's life science programs (Figure 1). Not long after the inauguration of NASA, the United States' first space station, Skylab, was launched in 1973 aboard a Saturn V rocket. The goal of Skylab was to serve as a laboratory environment for a variety of experiments spanning the fields of solar physics, Earth sciences, medicine, materials processing, and biology [7]. Many of the biology experiments focused on crew health and human physiology, i.e., validating instruments for measurements of mass in the absence of gravity and performing cytogenetic studies of blood [7]. However, there was also an early interest in microbial studies. For example, sidelined by larger experiments was a student-proposed study investigating the viability, growth rates, and morphology 
of dormant microbes and spores in microgravity [8]. Although rudimentary and simplistic in its use of hardware, this experiment was an example that hinted at the future capabilities and advances in technology of microbial studies in space.

Another iteration of life science missions came with the birth of the Space Shuttle Program in the 1980s. Aboard the Columbia shuttle launch in 1996 was the Life and Microgravity Spacelab, containing 16 life science experiments, with a primary focus on human life sciences and animal models [9]. Although not particularly advancing technologies for investigations of microorganisms, the Spacelab missions were fundamental in setting up the infrastructure for the International Space Station (ISS), which is now a key resource for microbiology research and associated technologies.

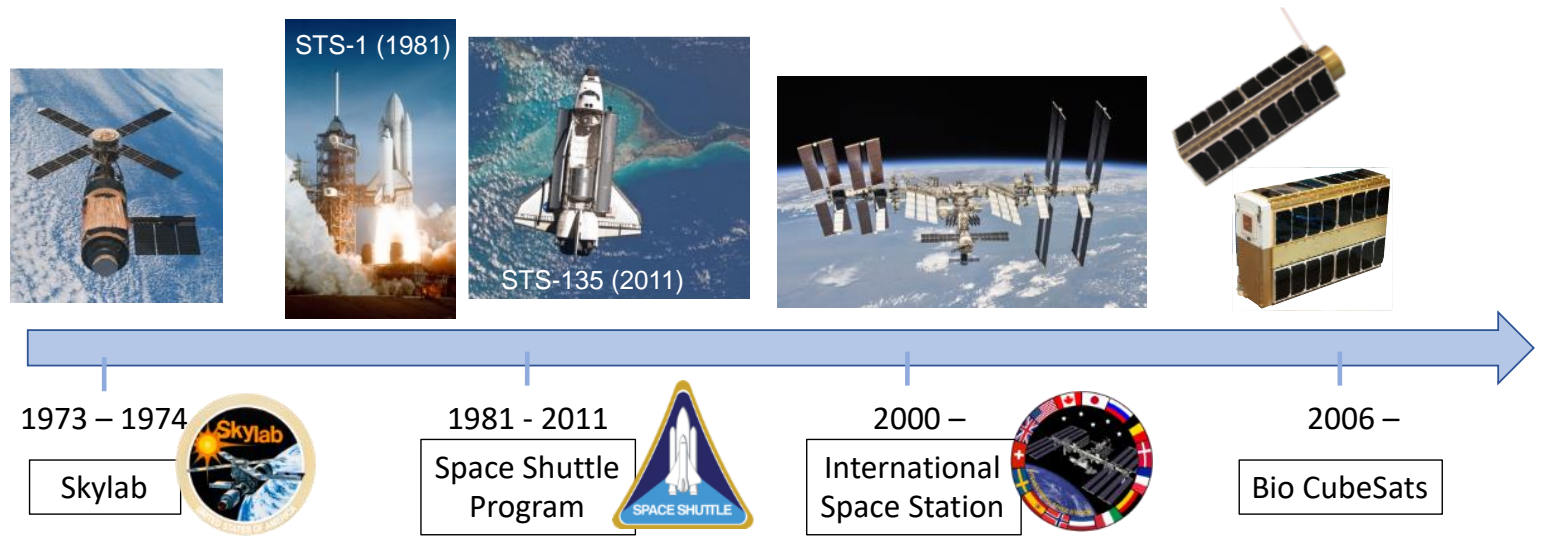

Figure 1. NASA's life science programs.

The ISS, over the course of its lifespan, has implemented over 40 facilities providing capabilities for life sciences research. Among these facilities are key technologies for conducting studies on microorganisms. In particular, there is an increasing prevalence of automated systems, such as the Mobile Spacelab, its subsidiary BioChip Spacelab, and CellCult, among many others. Some of the automated technologies in these facilities include microfluidics, various microscopy techniques, bioreactors, and multi-sample collection systems, all of which are key for biological experiments [1012]. Furthermore, the automation of these technologies on the ISS, where crew members are present to assist with biological experiments, is a key indicator of the importance in self-sustaining experiments for biological research beyond LEO. However, before this extension can take place, the next key step is miniaturizing and converting these technologies to systems independent of a larger facility like the ISS.

\section{Biological CubeSat Missions}

In 2006, NASA Ames Research Center pioneered a new era of biological studies and technology development in space with the launch of GeneSat-1, the first fully automated and self-contained biological CubeSat to go to space. GeneSat-1 employed some of the fundamental capabilities of the ISS facilities discussed previously-microfluidics and cell growth detection systems-contained within a free-flying, $3 \mathrm{U}(1 \mathrm{U}=10-\mathrm{cm}$ cube) platform to study gene expression in LEO [13]. From there, NASA Ames developed five additional free-flying biological CubeSats, each building on the previous CubeSat's infrastructure. An overview of each nanosatellite can be seen in Table 1. PharmaSat launched in 2009 and utilized a three-LED optical sensor to monitor microbial activity, this time testing yeast cells and their response to a fungicide in microgravity [14]. In 2010, Organism/Organic Exposure to Orbital Stresses (O/OREOS) successfully integrated two independent astrobiology studies in one CubeSat [15]. O/OREOS Space Environment Survivability of Live Organisms (SESLO) studied the ability of bacteria to adapt to the stresses of the space environment, while O/OREOS Space Environment Viability of Organics (SEVO) monitored the stability and changes in different organic molecules [15]. Four years later, SporeSat was launched, employing unique lab-on-a-chip devices termed biology compact discs (bioCDs). These devices utilized ion-sensitive electrodes to measure 
concentrations of calcium in fern spores and were rotated to simulate artificial gravity using miniaturized centrifuges, validating novel CubeSat technologies for biological experiments in space [16]. EcAMSat launched in 2017 as the largest biological nanosatellite thus far, and as the first $6 \mathrm{U}$ CubeSat to be deployed from the ISS [5]. Its main objective was to study antibiotic resistance in a pathogenic bacterium. The microfluidics and infrastructure used for all these LEO missions would set up the technological framework for the next and most recent mission.

The newest nanosatellite in the succession of NASA Ames' biological CubeSat program is BioSentinel. BioSentinel is the first CubeSat designed to perform biological experiments in interplanetary deep space and is planned to launch as the sole biological secondary payload on NASA's Artemis-1 rocket [17-19]. After deployment and a $700-\mathrm{km}$ lunar fly-by, BioSentinel will reach a stable heliocentric orbit and perform experiments for a minimum of 6 months. The primary goal of the mission is to investigate the DNA damage response to the deep space environment in the budding yeast $S$. cerevisiae. BioSentinel is a highly sophisticated and automated $6 \mathrm{U}$ CubeSat equipped with a series of subsystems designed and developed for the deep space environment, including solar panel arrays, batteries, star tracker and micro-propulsion navigation systems, transponder, antennas, and command and data handling systems, all of them occupying approximately $2 \mathrm{U}$ of the spacecraft [18]. The remaining $4 \mathrm{U}$ volume is occupied by the BioSensor payload, which contains all the instruments required to support the biological experiments. The BioSensor also contains a Timepixbased linear energy transfer (LET) spectrometer for radiation dose measurements and particle characterization. The microfluidics system is composed of 18 fluidic cards with 16 microwell per card (total of 288 microwells) [18]. Once in space, desiccated yeast cells will be rehydrated by injection of a mixture of growth medium and a metabolic indicator dye. Cell growth and metabolic activity will be monitored using an optical detection system consisting of three different LED lights and a TAOS light-to-voltage optical detector per well [19]. Each fluidic card also has a dedicated thermal control system that allows it to maintain the yeast cells in a benign cold environment until activation at a higher temperature. All the data will be telemetered back to Earth via the deep space network (DSN). In addition to the deep space mission, an identical copy of the BioSensor payload will be flown on the ISS, allowing for biological comparisons in deep space and LEO. Importantly, BioSentinel builds and improves upon proven technologies flown in previous biological CubeSats and, at the same time, provides a new platform for the space research community to conduct future missions using different organisms and novel instruments.

Table 1. NASA's biological CubeSat missions.

\begin{tabular}{cccl}
\hline $\begin{array}{c}\text { CubeSat Mission } \\
\text { (Size; Launch) }\end{array}$ & $\begin{array}{c}\text { Biological } \\
\text { Study }\end{array}$ & Research Investigation & \multicolumn{1}{c}{ Technology Development } \\
\hline GeneSat-1 & E. coli & Microgravity effects on & - 12-well fluidic card \\
(3U; 2006) & (bacterium) & gene expression & - LED optical detection \\
PharmaSat & S. cerevisiae & Microgravity effects on & - 48-well fluidic card \\
(3U; 2009) & (yeast) & antifungal response & - 3 LED optical sensor \\
O/OREOS SESLO & B. subtilis & Microgravity \& LEO & - 3 LED optical sensor \\
(3U; 2010) & (bacterium) & radiation effects & - Multiple time point activation \\
SporeSat & C. richardii & Microgravity effects on & - Mini centrifuges in space \\
(3U; 2014) & (fern spores) & calcium transport & - Lab-on-a-chip devices (BioCDs) \\
EcAMSat & E. coli & Microgravity effects on & - 48-well fluidic card \\
(6U; 2017) & (uropathogenic) & antibiotic response & - LED optical sensor \\
& & & - Variable dose delivery \\
BioSentinel & S. cerevisiae & Deep space radiation & - 288 wells in 18 fluidic cards with \\
dedicated thermal control \\
(6U; 2021/2022) & & effects & - LET spectrometer \\
& & & - ISS control experiment \\
\hline
\end{tabular}




\section{Future Technologies and Conclusions}

As highlighted in the preceding sections, technology continues to evolve as humanity once again prepares to embark upon deep space missions. These technologies enable more biological missions to be performed, building fundamental knowledge to answer the inquiries regarding biology's response to the space environment. Currently, several novel biosensor technologies for space applications are under development. One example takes advantage of a common technique used in industrial fermentation processes, called dielectric spectroscopy, by measuring changes in cell physiology via changes in capacitance. This indicates potential for new sensors to be implemented onboard BioSentinel's foundation, or to be implemented onto a different framework for different mission conditions. Other commercial technologies, such as miniaturized DNA/RNA sequencers, may be further developed for use in space for mutagenesis and gene expression studies. Other areas of interest, such as multigeneration and adaptive evolution studies and research in microbial communities and biofilms, also house potential for future mission consideration and technological developments.

Although research in space biology has been done for decades, there is still much to do. By building upon the history and technologies of the past, it will be possible to move forward confidently and safely into the next era of human space exploration.

Author Contributions: E.M.H. and A.K. contributed equally to this work. E.M.H., A.K. and S.R.S. conceptualized and wrote this paper. All authors have read and agreed to the published version of the manuscript.

Funding: The BioSentinel mission is supported by the Advanced Exploration Systems Program Office at NASA Headquarters.

Acknowledgments: We thank past and present members of the NASA BioSentinel mission and the Space Life Sciences Training Program (SLSTP).

Conflicts of Interest: The authors declare no conflict of interest.

\section{References}

1. Bizzarri, M.; Monici, M.; van Loon, J.J.W.A. How Microgravity Affects the Biology of Living Systems. BiomMed Res. Int. 2015, 2015, 1-4, doi:10.1155/2015/863075.

2. Ferl, R.J.; Paul, A.-L. The effect of spaceflight on the gravity-sensing auxin gradient of roots: GFP reporter gene microscopy on orbit. NPJ Microgravity 2016, 2, doi:10.1038/npjmgrav.2015.23.

3. Taylor, P.W. Impact of space flight on bacterial virulence and antibiotic susceptibility. Infect. Drug Resist. 2015, 8, 249-262, doi:10.2147/IDR.S67275.

4. Furukawa, S.; Nagamatsu, A.; Nenoi, M.; Fujimori, A.; Kakinuma, S.; Katsube, T.; Wang, B.; Tsuruoka, C.; Shirai, T.; Nakamura, A.J.; et al. Space Radiation Biology for “Living in Space." BioMed Res. Int. 2020, 2020, doi:10.1155/2020/4703286.

5. Padgen, M.R.; Chinn, T.N.; Friedericks, C.R.; Lera, M.P.; Chin, M.; Parra, M.P.; Piccini, M.E.; Ricco, A.J.; Spremo, S.M. The EcAMSat fluidic system to study antibiotic resistance in low earth orbit: Development and lessons learned from space flight. Acta Astronaut. 2020, 173, 449-459, doi:10.1016/j.actaastro.2020.02.031.

6. Diaz-Aguado, M.F.; Ghassemieh, S.; Van Outryve, C.; Beasley, C.; Schooley, A. Small Class-D spacecraft thermal design, test and analysis - PharmaSat biological experiment. In Proceedings of the 2009 IEEE Aerospace Conference, Big Sky, MT, USA, 7-14 March 2009; pp. 1-9, doi:10.1109/AERO.2009.4839352.

7. NASA Technical Reports Server (NTRS). Available online: https://ntrs.nasa.gov/citations/19780017172 (accessed on 14 October 2020).

8. NASA Technical Reports Server (NTRS). Available online: https://ntrs.nasa.gov/citations/19740025164 (accessed on 14 October 2020).

9. NASA Technical Reports Server (NTRS). Available online: https://ntrs.nasa.gov/citations/19980206462 (accessed on 14 October 2020). 
10. Mobile SpaceLab.

Available

online: https://www.nasa.gov/mission_pages/station/research/experiments/explorer/Facility.html?\#id=7692 (accessed on 14 October 2020).

11. BioChip SpaceLab. https://www.nasa.gov/mission_pages/station/research/experiments/explorer/Facility.html?\#id=7666 (accessed on 14 October 2020).

12. CellCult. Available online: https://www.nasa.gov/mission_pages/station/research/experiments/explorer/Facility.html?\#id=377 (accessed on 14 October 2020).

13. Ricco, A.J.; Hines, J.W.; Piccini, M.; Parra, M.; Timucin, L.; Barker, V.; Storment, C.; Friedericks, C.; Agasid, E.; Beasley, C.; et al. Autonomous genetic analysis system to study space effects on microorganisms: Results from orbit. In Proceedings of the TRANSDUCERS 2007-2007 International Solid-State Sensors, Actuators and Microsystems Conference, Lyon, France, 10-14 June 2007; pp. 33-37, doi:10.1109/SENSOR.2007.4300065.

14. Ricco, A.J.; Parra, M.; Niesel, D.; Piccini, M.; Ly, D.; McGinnis, M.; Kudlicki, A.; Hines, J.W.; Timucin, L.; Beasley, C.; et al. PharmaSat: Drug dose response in microgravity from a free-flying integrated biofluidic/optical culture-and-analysis satellite. In Proceedings of the SPIE 7929, Microfluidics, BioMEMS, and Medical Microsystems IX, San Francisco, CA, USA, 14 February 2011; doi:10.1117/12.881082.

15. Nicholson, W.L.; Ricco, A.J.; Agasid, E.; Beasley, C.; Diaz-Aguado, M.; Ehrenfreund, P.; Friedericks, C.; Ghassemieh, S.; Henschke, M.; Hines, J.W.; et al. The O/OREOS mission: First science data from the Space Environment Survivability of Living Organisms (SESLO) payload. Astrobiology 2011, 11, 951-958, doi:10.1089/ast.2011.0714.

16. Salim, W.W.A.W.; Park, J.; Rickus, J.L.; Rademacher, A.; Ricco, A.J.; Schooley, A.; Benton, J.; Wickizer, B.; Martinez, A.; Mai, N.; et al. SporeSat: A nanosatellite platform lab-on-a-chip system for investigating gravity threshold of fern-spore single-cell calcium ion currents. In Proceedings of the Solid-State Sensors, Actuators and Microsystems Workshop, Hilton Head Island, SC, USA, 8-12 June 2014; pp. 111-114, doi:10.31438/trf.hh2014.32.

17. Massaro Tieze, S.; Liddell, L.C.; Santa Maria, S.R.; Bhattacharya, S. BioSentinel: A Biological CubeSat for Deep Space Exploration. Astrobiology 2020, 20, doi:10.1089/ast.2019.2068.

18. Ricco, A.J.; Santa Maria, S.R.; Hanel, R.P.; Bhattacharya, S. BioSentinel: A 6U Nanosatellite for Deep-Space Biological Science. IEEE Aerosp. Electron. Syst. Mag. 2020, 35, 6-18, doi:10.1109/MAES.2019.2953760.

19. Santa Maria, S.R.; Marina, D.B.; Massaro Tieze, S.; Liddell, L.C.; Bhattacharya, S. BioSentinel: Long-Term Saccharomyces cerevisiae Preservation for a Deep Space Biosensor Mission. Astrobiology 2020, 20, doi:10.1089/ast.2019.2073.

Publisher's Note: MDPI stays neutral with regard to jurisdictional claims in published maps and institutional affiliations.

(C) 2020 by the authors. Submitted for possible open access publication under the terms and conditions of the Creative Commons Attribution (CC BY) license (http://creativecommons.org/licenses/by/4.0/). 